\title{
BIOCHEMICAL STUDIES ON PHENYLHYDRAZINE INDUCED EXPERIMENTAL ANEMIC ALBINO RATS
}

Nirjala Laxmi Madhikarmi ${ }^{1}$, Kora Rudraiah Siddalinga Murthy ${ }^{2}$

\section{ABSTRACT}

INTRODUCTION: The present study evaluated the modulatory effects of diphenylhydrazine induced experimental wistar albino rats and also to assess various biochemical parameters in whole blood and red blood cell lysate.

MATERIALAND METHODS: Twenty male albino rats weighing 180-200 gm were selected for the study and divided in two groups; ten phenylhydrazine dihydrochloride (PHZ) induced anemia and ten healthy control. Thiobarbituric acid reactive substances and lipid hydroperoxide were measured as lipid peroxidation parameter. The antioxidant vitamins $\mathrm{A}, \mathrm{C}$ and $\mathrm{E}$ and enzymatic antioxidants; catalase, glutathione peroxidase and superoxide dismutase were also assessed.

RESULTS: Phenylhydrazine induced anemic rats showed a significant increase in the lipid peroxidation and decrease in the antioxidants as compared to healthy rats.

CONCLUSION: The study concludes that phenylhydrazine induced experimental anemic albino rats showed increased oxidative stress than compared with healthy albino rats.

KEYWORDS: Albino rats; Anemia; Antioxidant; Lipid peroxidation; Oxidative stress; Phenylhydrazine

1. Assistant Professor, Department of Biochemistry, M. B. Kedia Dental College, Birgunj, Nepal

2. Professor, Department of Biochemistry, Central College Campus, Bangalore University, India

\author{
For Correspondence \\ Dr. Nirjala Laxmi Madhikarmi \\ Assistant Professor, \\ Department of Biochemistry, \\ M. B. Kedia Dental College, \\ Birgunj, Nepal \\ E-mail:nirjala4@gmail.com
}




\section{INTRODUCTION}

Anemia is one of the most widespread disorders of blood which affect the populations of all ages throughout the world. It is a public health problem that affects populations both rich and poor countries. It is a pathologic condition, in which there is a decrease in red blood cell mass or a decrease in the amount of $\mathrm{Hb}$. Further, iron deficiency affects the production of other proteins containing $\mathrm{Fe}^{2+}$, such as cytochromes, myoglobin, catalase and peroxidases. Worldwide at any given moment, more individuals have iron deficiency anemia than any other health problem. Non-communicable diseases kill more than 36 million people each year. Mostly a bane of middle and low income countries, they share four risk factors: tobacco use, physical inactivity, the harmful use of alcohol and unhealthy diets. $^{1,2}$

However, the incidence of this disorder is higher in the developing countries than in the developed countries ${ }^{1}$ due to poverty and lack of hygiene. The situation is aggravated by factors such as nutritional deficiencies and high prevalence of parasitic gastrointestinal infections which cause loss of blood. Number of other conditions, such as malaria and haemoglobinopathies are also responsible, often in combination $^{2,3}$. Wistar albino rats (Rattus norvegicus) is one of the most commonly used animals in biomedical research. Over the years, rats have been used in many experimental studies, which have added to our understanding of genetics, diseases, the effects of drugs, and other topics in health and medicine. Animal research holds the key for solution to AIDS, cancer, heart disease, aging, congenital defects and also healthier, longer and better life with much less pain and suffering for humans. ${ }^{4}$

Lipid peroxidation has been identified as a basic deteriorative reaction in the cellular mechanism of the disease and is initiated by the free radicals which oxidize the PUFAs leading to the formation of conjugate dienes ultimately resulting in the production of hydroperoxides, cyclic peroxides and malondialdehyde. Acting as the first line of defense against the production of such hydroperoxides is a naturally occurring antioxidant glutathione, which is a major source of free thiol in most living cells. GSH also participates in diverse biological processes such as detoxification of xenobiotics and modulation of enzyme activity by disulphide interchange. Alterations in the normal GSH status influence the normal redox status of the cell and hence may lead to lipid peroxidation $^{5-7}$.

\section{MATERIALAND METHODS}

Adult male Albino rats were selected for animal experiment. The animals were housed in a stainless steel cage ( 2 animals per cage) and acclimatized with free access to tap water and standard diet at controlled temperature $\left(22-24^{\circ} \mathrm{C}\right)$ and humidity (50 60\%), on a $12 \mathrm{hr}$ dark / light cycle for at least one week before the experiments. The acclimatized rats weighing 150 - $200 \mathrm{~g}$ were purchased from Shri Raghavendra Animal House, Vijaya Nagar, Bangalore, India. All experiments on rats were carried out in absolute compliance with the ethical guidelines for care and use of laboratory animals. All animal procedures were reviewed and approved by the Institutional Animal Care and Use Committee, and Committee for the Purpose of Control and Supervision of Experiments on Animals (CPCSEA), New Delhi, India. All the chemicals and reagents used during experimentation were of analytical grade.

The vitamin mixture composition according to Williams and Mills $(1970)^{8}(\%)$ : vitamin A acetate, 0.18; vitamin $\mathrm{D}_{2}$, 0.0125; DL $\alpha$ tocopherol acetate, 2.2; ascorbic acid, 4.5; inositol, 0.5; choline chloride, 7.5; menadione, 0.225 ; paminobenzoic acid, 0.5; niacin, 0.425; riboflavin, 0.1; thiamine hydrochloride, 0.1 ; calcium pantothenate, 0.3; biotin, 0.002; folic acid, 0.009; vitamin $\mathrm{B}_{12}, 0.000135$.

The salt mixture composition according to Hubbel et al $(1937)^{9}(\%)$ : Calcium carbonate, 6.86; calcium citrate, 30.83; calcium phosphate monobasic, 11.28; manganese carbonate, 3.52; magnesium sulfate, 3.83 ; potassium chloride, 12.47 ; dipotassium phosphate, 21.88; sodium chloride, 7.71; copper sulfate, 0.00777; manganese sulfate, 0.02008; potassium aluminum sulfate, 0.00923; potassium iodide, 0.00405; sodium fluoride, 0.05070 .

The normal diet contained; casein $210 \mathrm{~g}$, starch $640 \mathrm{~g}$, ground nut oil $80 \mathrm{ml}$, salt mixture $40 \mathrm{~g}$, vitamin mixture $30 \mathrm{~g}$ and $390 \mathrm{mg} \mathrm{FeSO}_{4}$. $7 \mathrm{H}_{2} \mathrm{O}$. Iron deficient diet was prepared by excluding $\mathrm{FeSO}_{4} \cdot 7 \mathrm{H}_{2} \mathrm{O}$ and the amount was replaced by starch. Control wistar albino rats fed on normal standard laboratory diet (10 animals). Anemia was induced in experimental animals by intraperitoneal (IP) injection of phenylhydrazine dihydrochloride (PHZ, 10mg/kg weight) and was fed on iron deficient diet for 15 days. The animals were sacrificed on the $16^{\text {th }}$ day by cervical dislocation.

Blood ( $3 \mathrm{ml})$ was collected from posterior venacava in tubes containing ethylenediamine tetraacetic acid (EDTA) for analysis. Plasma was collected by centrifuging blood 
containing anticoagulant at $4000 \mathrm{rpm}$ for $10 \mathrm{~min}$, carefully separated and was stored in a sterile and dry vial for analysis. After plasma separation, erythrocyte pellet was washed thrice with chilled physiological saline $(0.01 \mathrm{M}$ phosphate buffer saline, PBS; pH 7.45 containing $0.85 \%$ sodium chloride); $0.5 \mathrm{ml}$ of cell suspension was diluted with $2 \mathrm{ml}$ cold distilled water to lyse the erythrocytes. The lysed RBC was again centrifuged at $4000 \mathrm{rpm}$ for $10 \mathrm{~min}$ to get clear supernatant (RBC hemolysate) and the pellet was discarded.

The hemoglobin level in whole blood (EDTA) was determined by the method of Cyanmethemoglobin using Drabkin's solution. Serum iron and total iron binding capacity (TIBC) was determined by Ferrozine method. The lipid peroxidation product, malondialdehyde (MDA) was determined by the method of Buege and Aust (1978) ${ }^{10}$. The malondialdehyde concentration in RBC lysate was determined by the method of Donnam (1950) ". The lipid hydroperoxide (LPHO) was determined according to Jiang (1992) ${ }^{12}$ using FOX reagent. The nitrite and nitrate concentration was determined using reduced cadmium by the method of Cortas and Wakid $(1990)^{13}$.The nitrate was reduced to nitrite by activated cadmium. Nitrite was determined by diazotization of sulfanilamide and coupling to naphthylethylene diamine according to Cortas and Wakid (1990).

Enzymatic Antioxidant Assays: Superoxide dismutase $(\mathrm{Cu} / \mathrm{Zn}-\mathrm{SOD})$ activity in hemolysed $\mathrm{RBC}$ and various tissues homogenate were determined by the method of Kakkar et al $(1984)^{14}$. Catalase (CAT) activity in the whole blood and hemolysed RBC (erythrocytes) were assayed in the erythrocyte lysates by the method of Sinha $(1972)^{15}$. Glutathione peroxidase (GPx) activity was analyzed in hemolysed RBC lysates and various tissues homogenate by the method of Rotruck $(1973)^{16}$. Determination of NonEnzymatic Antioxidant: Vitamin A was estimated by the method of Bessey (1946) $)^{17}$. Vitamin C (Ascorbic acid) was estimated by the method of Natelson $(1971)^{18}$. Vitamin E was estimated by the method of Baker and Frank $(1968)^{19}$. Total antioxidant activity (TAA) was performed according to Benzie and Strain (1996) ${ }^{20}$. Glutathione reduced (GSH) was determined according to the method of Beutler and Kelley $(1963)^{21}$. The total protein was estimated by the method of Lowry $(1951)^{22}$ using Folin-Ciocalteau Reagent. Erythrocyte fragility test was carried out by the method of Dacie and Lewis $(1968)^{23}$. The packaged program SPSS (Statistical package for social sciences) for windows version 13.0 (SPSS, Chicago, Il, USA) was used for statistical analysis.

\section{RESULTS}

Free radicals are substances that easily react with biomolecules and also responsible for the pathogenesis of several diseases including anemia. Oxygen derivatives, which constitute a large portion of FRs, give rise to reversible or irreversible damages to nucleic acids, proteins, amino acids, lipids, carbohydrates and connective tissue macromolecules. A direct correlation between anemia and oxidative stress is shown before by many researchers. In this study, we aimed to investigate correlation between free radicals and antioxidants in healthy and anemic rats. For this purpose SOD, CAT, GPX, LPHO, TBARS, VIT A, $\mathrm{C}$ and $\mathrm{E}$ and TAA levels were estimated in healthy and induced anemic rats. On analysis, after injection of phenylhydrazine dihydrochloride $(10 \mathrm{mg} / \mathrm{kg}$ body weight) haemoglobin levels in experimental rats were highly decreased as shown in Table 1: $(p<0.005$, the level of significance) when compared to healthy animals.

Table 1: Lipid peroxidation and enzymatic antioxidants parameters of albino rats

\begin{tabular}{|c|c|c|c|c|}
\hline \multirow{2}{*}{ Variables } & \multicolumn{2}{|c|}{ Whole blood } & \multicolumn{2}{|c|}{ Red Blood Cell lysate } \\
\hline & Control & Anemia & Control & Anemia \\
\hline $\mathrm{Hb}(\mathrm{g} / \mathrm{dl})$ & $14.21 \pm 1.32$ & $5.6 \pm 0.89^{*}$ & - & - \\
\hline \multicolumn{5}{|c|}{ Lipid peroxidation parameters: } \\
\hline TBARS $(\mathrm{nmol} / \mathrm{ml})$ & $3.03 \pm 1.50$ & $6.04 \pm 0.14$ & $2.19 \pm 0.35$ & $3.18 \pm 0.29$ \\
\hline LPHO (nmol/ml) & $1214.45 \pm 30.18$ & $1591.87 \pm 41.60$ & $478.35 \pm 20.85$ & $4958.23 \pm 26.88^{*}$ \\
\hline $\mathrm{NO}_{2}(\mu \mathrm{mol} / \mathrm{l})$ & $380.48 \pm 2.09$ & $1076.48 \pm 52.31^{*}$ & $50.26 \pm 4.05$ & $201.37 \pm 15.56^{* *}$ \\
\hline $\mathrm{NO}_{3}(\mu \mathrm{mol} / \mathrm{l})$ & $6523.38 \pm 29.8$ & $7869.44 \pm 10.03$ & $1477.8 \pm 21.43$ & $2166.80 \pm 22.41$ \\
\hline \multicolumn{5}{|c|}{ Non-enzymatic Antioxidants parameters : } \\
\hline $\mathrm{GSH}(\mathrm{mg} / \mathrm{dl})$ & $60.47 \pm 12.60$ & $33.66 \pm 2.84 *$ & - & - \\
\hline $\operatorname{VIT~A}(\mu \mathrm{g} / \mathrm{dl})$ & $2682.21 \pm 15.0$ & $3571.44 \pm 16.48^{* *}$ & $438.11 \pm 16.49$ & $452.43 \pm 11.61^{* *}$ \\
\hline VIT C (mg/dl) & $17.23 \pm 0.12$ & $9.50 \pm 0.74^{*}$ & $9.18 \pm 1.52$ & $2.31 \pm 0.47$ \\
\hline $\operatorname{VIT~E}(\mathrm{mg} / \mathrm{dl})$ & $88.56 \pm 0.45$ & $33.6 \pm 0.23^{* *}$ & $43.23 \pm 0.26$ & $153.58 \pm 0.41^{* *}$ \\
\hline $\mathrm{TAA}(\mu \mathrm{mol} / \mathrm{l})$ & $51.94 \pm 0.58$ & $30.29 \pm 5.062^{* *}$ & $4.08 \pm 0.75$ & $9.84 \pm 5.17^{* *}$ \\
\hline $\mathrm{TP}(\mathrm{mg} / \mathrm{dl})$ & $87.61 \pm 5.83$ & $40.63 \pm 9.24 * *$ & $48.84 \pm 1.63$ & $56.30 \pm 3.50$ \\
\hline \multicolumn{5}{|c|}{ Enzymatic Antioxidants parameters : } \\
\hline $\mathrm{CAT}(\mathrm{U} / \mathrm{mgHb})$ & $424.71 \pm 13.46$ & $873.39 \pm 25.46^{* *}$ & $150.16 \pm 5.29$ & $289.40 \pm 14.39$ \\
\hline $\mathrm{SOD}(\mathrm{U} / \mathrm{gHb})$ & - & - & $21.66 \pm 1.84$ & $93.67 \pm 3.64^{*}$ \\
\hline $\mathrm{GPx}(\mathrm{U} / \mathrm{gHb})$ & - & - & $2.71 \pm 1.03$ & $7.91 \pm 0.05^{* *}$ \\
\hline
\end{tabular}

Note : Hb-Hemoglobin, TBARS-thiobarbituric acid reactive substances, LPHO-lipid hydroperoxides, $\mathrm{NO}_{2}$-nitrite, $\mathrm{NO}_{3}$ nitrate, GSH-glutathione, VIT A-vitamin A, VIT C-vitamin C, VIT E-vitamin E, TAA-total antioxidant activity, TP-total protein, CAT-catalase, SOD-superoxide dismutase, GPxglutathione peroxidase, (Level of significance- ${ }^{*} \mathrm{p}<0.005$, $* * \mathrm{p}<0.05)$

The TBARS levels were increased in experimental anemic rats both in whole blood and RBC lysates but the increase was not statistically significant whereas, increase in LPHO levels were increased both in whole blood and RBC lysates but it was 
significant only in RBC lysates. Nitrite level was increased both in whole blood and RBC lysates but the increase in nitrate was not significant. Non-enzymatic antioxidants were statistically significant in whole blood sample in anemic rats whereas, only few parameters showed significance in RBC lysates. The enzymatic antioxidant, catalase was significant only in whole blood at $\mathrm{p}<0.05$. Superoxide dismutase and glutathione peroxidase was statistically significant in RBC lysates. On osmotic fragility test carried out in control and PHZ induced anemic rats (Fig I :) showed increasd hemolysis in PHZ treated albino rats as compared to healthy controls.

\section{DISCUSSION}

Testing drugs in animals before doing so in human helps researchers find potential toxic side effects, as well as understand the metabolism of drug compounds and consequent effects seen throughout the body. It is well known since long time that carbohydrates, proteins and lipids are biologically active macromolecules involved in various metabolic and energy yielding processes of cellular systems. Metabolic disturbances of any of these molecules are known to contribute to several chronic pathological states. Amino acids, free as well as in the form of constituents of proteins, play an important role in maintaining the structural integrity and folding nature of proteins and any imbalance or inadequacy of these components influence protein synthesis, thereby affecting the protein turnover. Free radical mediated lipid peroxidation is involved in many pathological processes and biological systems possess self-defensive mechanism against these peroxides mediated through enzymatic and nonenzymatic systems. The use of animals in research is prevalent because they share at least 200 common illnesses and diseases with humans. ${ }^{4,24}$

The $\mathrm{RBC}$ are intrinsically prone to oxidative stress because they are exposed to high oxygen tension, and have a characteristic structural composition with polyunsaturated fatty acid in the membrane, besides the presence of haemoglobin bound iron. However, membrane and cytoplasmic compartments of $\mathrm{RBC}$ have an efficient antioxidant mechanism that maintains their integrity. A detoxifying system consists of reduced glutathione (GSH), SOD, CAT. GPx and Vit E prevent oxidative damage. In addition, there is also a system consisting of NADPHdependent methemoglobin reductase, ascorbic acid, glutathione reductase (GR), whose main role is the repairing of damage that follows oxidative stress. ${ }^{25}$ In normal physiological state, oxidants generated during metabolism play a significant role in maintaining oxidant-antioxidant ratio. In pathological state, an increase in the reactive free radicals creates an imbalance in this ratio thereby making macromolecules vulnerable to oxidative damage. As a result, proteins undergo rapid oxidation leading to the alterations in their structural integrity and in assessing oxidative damage lipid peroxidation and protein oxidation are generally used as biomarkers. $^{26}$

Lipid peroxidation has been identified as a basic deteriorative reaction in the cellular mechanism of the anemia and is initiated by the free radicals which oxidize PUFAs leading to the formation of conjugate dienes ultimately resulting in the production of hydroperoxides, cyclic peroxides and malondialdehyde. Acting as the first line of defence against the production of such hydroperoxides is a naturally occurring antioxidant glutathione (GSH), a major source of free thiol in most living cells. GSH in addition also participates in diverse biological processes such as the detoxification of xenobiotics and modulation of enzyme activity by disulfide interchange. ${ }^{27}$ However, red cells have a potent antioxidant protection that modifies GSH, GPx, Vitamins A, C and E. Synergistic and cooperative interactions of these antioxidants rely on the sequential degradation of peroxides and free radicals as well as on mutual protections of enzymes. Oxidative stress arises when there is an imbalance between radical generating and radical scavenging activity; it may therefore increase formation of oxidation products. Oxidation of erythrocytes induces membrane injury, methemoglobin formation and eventually destruction of the cell. Lipids especially PUFAs are sensitive to oxidation, leading to the term lipid peroxidation, of which, MDA is the most abundant. The accumulation of MDA in tissues or biological fluids is indicative of the extent of free radical generation, OS and tissue damage. ${ }^{28}$

All cells contain iron which is of fundamental biological importance, being a micronutrient that is active in oxidative metabolism, cell growth and reproduction, as well as in oxygen transportation (hemoglobin) and storage (myoglobin). Nevertheless, levels of iron are not always sufficient, because they are influenced by physiological and dietary factors. Oral administration of $10 \mathrm{mg} / \mathrm{kg}$ phenylhydrazine for 8 days reduced hematological indices by $50 \%$. PHZ altered the function of RBC by hemolysis characterized by $76.06 \%$ decrease in RBC, $46.27 \%$ decrease in $\mathrm{Hb}$ concentration, $65.24 \%$ decrease in $\mathrm{WBC}$ and $42.68 \%$ decrease in $\mathrm{PCV}^{29-30}$

Markovic et al..$^{31}$ reported decreased GSH concentration 
followed by the accumulation of superoxide anion and hydrogen peroxide in the plasma in phenylhydrazine treated anemic rats. On the other hand there was no change in lipid peroxidation in either the plasma, or the RBC of PHZ treated rats. The high levels of ROS cause the elevation of Heinz body formation in the RBC of PHZ treated rats, indicating that proteins could be the main site of PHZ-induced damage of RBC. GSSG / 2 GSH ratio is significantly higher in the plasma of PHZ-treated rats, which indicates an inefficient metabolism of the glutathione system and could be one of the causes of ROS accumulation. Plasma of PHZ-treated rats is the main site of oxidative stress mainly because the glutathione antioxidative mechanisms are less efficient in the scavenging of ROS, but there is good protection of this mechanism against lipid peroxidation.

Feeding iron deficient diet $(35 \mathrm{mg}$ iron $/ \mathrm{kg}$ ) during gestation and or lactation results in significant decrease in non heme iron as early as in 7 days old pups showing a stage of early iron deficient which later entered into the stage of IDA as shown by reduced PCV and $\mathrm{Hb}$ levels. ${ }^{32}$ This iron deficiency did not show any effect on body and brain weights of developing rats. However, there was a significant reduction in the activities of brain glutamate dehydrogenase, glutamate adenine deaminase and GABA-transferases enzymes, associated with GABA metabolism, in gestationally and lactationally iron deficient pups. However, earlier reports have reported the involvement of iron in synthesis and packaging of neurotransmitters, their uptake and degradation into other iron containing proteins which may directly or indirectly alter brain function. Iron deficiency during developmental stage of brain may cause irreversible disturbances and damage to GABA neurotransmitters system. ${ }^{28-33}$

Feces of rat fed with iron fortified diet were darker than rats without iron fortified diet. Iron and calcium are known to enhance the qualities of blood, bones, teeth and blood formation and also of cardiac function. ${ }^{33}$ They also play a predominant role in oxygen and electron transport. Fortified diet leads to the development of good immune system in rats and in humans which contributes extensively to freedom from frequent sickness and illness, resulting in good health as a result of consumption of high quality food.

Increased TBARS in iron deficient group was reported by Hamed et al. (2010) ${ }^{7}$ than normal healthy controls. Supplementation of iron in the form of ferrous ammonium sulfate form freeze dried juice mixtures to iron deficient rats produced significant reduction of TBARS. Iron may induce
OS only if it is given to non iron deficient subjects or if it was in ferric form. Rats fed on both freeze dried fruit juice and ferrous ammonium sulfate showed decreased TBARS level as compared to those fed only ferrous ammonium sulfate diet. The presence of antioxidant component; ascorbic acid, carotene, $\alpha$-tocopherol, polyphenols and condensed tannins in fruit juices has a beneficial effect and results in decreased TBARS level.

Mittal $(2002)^{34}$ reported eight weeks of iron deficiency in rat did not significantly change the gross weights of brain and liver. There was no effect on hemoglobin and hematocrit. The non-heme iron in liver and brain decreased significantly. The activities of glutamate dehydrogenase, glutamic acid decarboxylase and GABA-transferase in brain were decreased. The effects of iron deficiency on the levels of iron, copper, zinc and magnesium in the brain, liver, kidney, heart and lungs of albino rats was investigated by Oladiji (2003). ${ }^{35}$

The effect of the iron deficient diet on iron absorption is shortlived. Exposure to the ordinary amounts of dietary iron in the standard diet after a 2-week period of iron deprivation reduces iron absorption to normal levels in 3 days. Even when dietary iron deficiency is extended over a period of 3-10 months, iron absorption returns to control levels after 5 days feeding of an iron-replete diet. The lack of dietary iron appears to be of little importance in the control of iron absorption of humans, in contrast to the rats . ${ }^{27,36}$ Rats are continuously growing and are presumably in positive iron balance; thus, their need for iron is comparatively greater than man's. Further, rats excrete iron much more rapidly than do humans. Rats lose $0.23 \%$ of their body iron per day, while humans lose $0.10 \%$ per day. Thus it takes man 23 days to excrete the same proportion of body iron the rat loses in 1 day. Extrapolation would indicate that man would thus have to be deprived of iron for 115 days to acquire a net iron deficit comparable to that which that rat acquires in 5 days of iron-deficient feeding. Iron absorption in rats is increased by dietary iron deprivation, erythropoiesis in the rat is unaffected by dietary iron deprivation that increases iron absorption by more than a factor of four, iron absorption is not increased in bled rats of an amount of iron equivalent to that lost in 5 days of iron derivation, these findings are compatible with the concept that iron-absorption is controlled by depletion of iron from a specific pool, separate from the hepatic and erythrocytic pools, iron absorption in human subjects was unaffected by dietary iron deprivation for 13 days. ${ }^{36-39}$

\section{CONCLUSION}


Phenylhydrazine induction causes destruction of red blood cells by oxidation stress and many joint changes at cellular level resulting in anemia. The study suggests that damage caused by induction of phenylhydrazine is more in whole blood than RBC. The induction of PHZ decreases antioxidant defense mechanism resulting in decreased antioxidant levels. Nevertheless, there is need for thorough understanding of the phenylhydrazine on various other tissues (brain, heart, kidney, liver, lungs, spleen, etc) and are yet to be studied.

\section{ACKNOWLEDGEMENT}

We thank Indian Council for Cultural Relations, Bangalore and Indian Embassy (Nepal) for providing the Silver Jubilee Scholarship.

\section{REFERENCES}

1. Ogbe RRJ, Adoga GI, Abu, AH. Anti-anaemia potentials of some plant extracts on phenyl hydrazine induced anaemia in rabbits. JMedicinal Plants Res 2010; 4 (8): 680 - 4.

2. Crawley J. Reducing the burden of anaemia in infants and young children in malariaendemic countries of Africa: from evidence to action. Am J Tropical Medicine and Hygiene 2004 ; 71 (Suppl. 2) : 25-34.

3. De Benoist B, McLean E, Egli I, Cogswell M. Worldwide prevalence of anaemia 19932005, WHO Global Database on Anemia. Geneva: World Health Organization; 2008. p. 21.

4. http://en.wikipedia.org/wiki/Laboratory_rat

5. Olanlokun JO. Protective influence of vitamin $E$ on the antioxidant defence system in the whole blood and liver of normal and alloxan-induced diabetic rats. Indian $J$ Clin Biochem 2008; 23(1) : 62-6.

6. Kanwar SS, Nehru B. Modulatory effects of N-acetylcysteine on cerebral cortex and cerebellum regions of ageing rat brain. Nutr Hosp 2007; 22(1) : 95-100.

7. Hamed TE, Mohamed DA, Afifi AA, Al-Okbi SY. Impact of intake of plant foods iron on the recovery from iron deficiency in rats. Medical J Islamic World Academy of Sciences 2010; 18 (1) $: 13-20$.deficiency in rats. Medical J Islamic World Academy of Sciences 2010; $18(1): 13-20$.

8. Williams R, Band-Mills CF. The experimental production of zinc deficiency in rat. BrJ Nutr 1970; 24 : 989-1003.

9. Hubbel RB, Mendel KB, Wakeman AJ. A new salt mixture for use in experimental diets. JNutr 1937; $14: 273-85$.

10. Buege JA, Aust SD. Microsomal lipid peroxidation. Methods Enzymol 1978; 52 : 302-10.

11. Donnan SK. The thiobarbituric acid test applied to tissues from rats treated in various ways. Biochem J 1950; 182: 415.

12. Jiang ZY, Hunt JV, Wolff SP. Ferrous ion oxidation in the presence of xylenol orange for detection of lipid hydroperoxides in low density lipoprotein. Anal Biochem 1992; 202 : 384-9.

13. Cortas NK, Wakid NW. Determination of inorganic nitrate in serum and urine by a kinetic cadmium-reduction method. Clin Chem 1990; 36: 14403.

14. Kakkar P, Das B, Vishwanathan PN. A modified spectrophotometric assay of superoxide dismutase. Ind $J$ Biochem Biophys 1984; 21 : 1302.

15. Sinha KA. Colorimetric assay of catalase. J Biochem 1972; 47 : 389-94.

16. Rotruck JT, Pope L, Ganther HE, Swanson AB. Selenium biochemical role as a component of glutathione peroxidase. Science 1973; 179: 588-90.

17. Bessey OA, Lowry OH, Brock MJ, Lopez JA. The determination of vitamin $A$ and carotene in small quantities of blood serum. $J$ Biol Chem 1946; 166: 177-88.

18. Natelson S. In Techniques in Clinical Biochemistry. (CC Thomas, USA) 1971; $162: 288$.

19. Baker H, Frank O. Determination of Vitamin E. In: Clinical vitaminology. USA. 1968; 172-6.

20. Benzie IFF, Strain JJ. The ferric reducing ability of plasma (FRAP) as a measure of "antioxidant power": The FRAP assay. Analytical Biochemistry 1996: 239; 70-6.

21. Beutler E, Duron O, Kelley BM. Improved method for the determination of blood glutathione. J Lab and Clin Med 1963; $61(5): 882-8$.

22. Lowry OH, Rosebrough NJ, Farr AL, Randall RJ. "Protein measurement with the Folin phenol reagent. J Biol Chem 1951; 193(1) : 265-75.

23. Dacie JV, Lewis SM. Practical haematology. New York: Grune \& Stratton Inc, $4^{\text {th }}$ edition. 1968: 173-7.

24. Nagababu E, Fabry ME, Nagel RL, Rifkind JM. Heme degradation and oxidative stress in murine models for hemoglobinopathies:thalassemia, sickle cell disease and hemoglobin Cdisease. Blood Cells Mol Dis 2008; 41(2) : 60-6. 
25. Ferreira ALA, Machado PEA, Matsubara LS. Lipid peroxidation, antioxidant enzymes and glutathione levels in human erythrocytes exposed to colloidal iron hydroxide in vitro. Brazilian JMed and Biol Res 1999; 32 : 689-94.

26. Kanth VR, Reddy PU, Raju TN. Behavioral, morphological and physiological shift in the rats administered with tryptophan deficient regimen. Nutr Hosp 2006; 21(5) : 596-603.

27. Kanwar S, Nehru B. Modulatory effects of N-acetylcysteine on cerebral cortex and cerebellum regions of ageing rat brain. Nutr Hosp 2007; 22(1) : 95-100.

28. Saleh MA, Al-Salahy, Bassam M; Sanousi, Samera A. Oxidative stress in blood of camels (Camelus dromedaries) naturally infected with Trypanosoma evansi. Vet Parasitol 2009; 162 : 192-9.

29. Agbor AG, Odetola AA. Haematological studies of Parquetina nigrescens on aemorrhagic anaemic rats. Afr J Med Sci 2001; $30: 105-9$.

30. Akah POA, Okolo CE, Ezike AC. The hematinic activity of the methanol leaf extract of Brillantasia nitens Lindau (Acanthaceae) in rats. African J Biotech 2009; 8(10) : 2389-93.

31. Markovic SD, Obradovic AD, Zizic JB, Ognianovic BI, Stain $A S$,Saicic ZS et al. Glutathione status in the blood rats after reticulosis induced by phenylhydrazine and bleeding. Arch Biol Sci Belgrade 2010; 62(3) : 589-94.
32. Batra J, Seth PK. Effect of iron deficiency on developing rat brain. Indian J Clin Biochem 2002; 17(2) : 108-14.

33. Ebuehi OAT, Oyewole AC. Biochemical studies of iron fortified Nigerian rice fed to phenylhydrazine induced anemic rats. Am J Biochem and Molecular Biology 2011; 1(2): 168-77.

34. Mittal RD, Pandey A, Mittal B, Agarwal K N. Effect of latent iron deficiency on GABA glutamate neuroreceptors in rat brain. Indian JClin Biochem 2002; 17(2) : 1-6.

35. Oladiji TA. Tissue levels of iron, copper, zinc and magnesium in iron deficient rats. Biokemistri 2003; 14 : 75-81.

36. Eisenstein RS. Iron regulatory proteins and the molecular control of mammalian iron metabolism. Annu Rev Nutr 2000; 20:627-62.

37. Aslan M, Horoz M, Kocyigit A, Ozgonul S, Celik H, Celik M, Erel O. Lymphocyte DNA damage and oxidative stress in patients with iron deficiency anemia. Mutation Res 2006; 601 : 144-9.

38. Matsumoto J, Mori N, Doi M, Kishida T, Ebihara K. Evaluation of iron bioavailability from bonito dark muscle using anemia rats. JAgric Food Chem 2003; 51 : 4478-82.

39. Walczewska A, Dziedzic B, Stepien T, Swiatek E, Nowak D. Effect of dietary fats on oxidative-antioxidative status of blood in rats. JClin Biochem Nutr 2010; 47: 18-26. 